\title{
DISTRIBUCIÓN DE FELINOS SILVESTRES EN ÁREAS PROTEGIDAS DE HONDURAS
}

\author{
Héctor Orlando Portillo Reyes ${ }^{1}$, Fausto Elvir ${ }^{2}$ \\ 1,2 Grupo de Investigadores de Biodiversidad para \\ Honduras (GIBH). \\ ${ }^{1}$ Fundación de Ciencias para el Estudio y la \\ Conservación de la Biodiversidad (INCEBIO) \\ Residencial Plaza 1era entrada BL-16, C 4606. \\ Tegucigalpa, Honduras
}

Autor de correspondencia: Héctor Orlando Portillo Reyes, hectorportilloreyes@gmail.com

\begin{abstract}
RESUMEN
En Honduras ocurren cinco especies de felinos, jaguar (Panthera onca), puma (Puma concolor), ocelote (Leopardus pardalis), caucel (Leopardus wiedii) y el yaguarundí (Puma yagouaroundi). Se comparó la composición de las cinco especies de felinos en 15 áreas naturales protegidas, las cuales se agruparon en tres regiones con diferentes zonas de vida, ecosistemas y gradientes altitudinal siendo estas; la región Moskitia, la región Central y la región Caribe. Se evaluó la detección de felinos con la presencia/ausencia en cada área. Se realizó un dendrograma y un análisis no métrico multidimensional para comparar la similitud entre áreas protegidas y especies. El hallazgo significativo es la ausencia del jaguar en las áreas protegidas de la región Central de Honduras, donde posiblemente se ha dado su extirpación. El puma, el ocelote, el caucel y el yaguarundí, se registran en las tres regiones del país. Al comparar la composición de los felinos en las tres regiones usando el análisis de similitud, este presentó diferencias significativas entre las áreas protegidas evaluadas. La región Central mostró menor composición que la región del Caribe y la Moskitia. La región de la Moskitia hondureña se muestra como la región con mayor resilencia para mantener las cinco especies de felinos.
\end{abstract}

Palabras clave: Composición, conglomerados, escalamiento multi-dimesional no métrico (NMDS), análisis de similitud (ANOSIM), disimilitud, Moskitia.

\footnotetext{
ABSTRACT

Honduras is territory of five species of cats, jaguar (Panthera onca), puma (Puma concolor), ocelots (Leopardus pardalis), margay (Leopardus wiedii) and the yaguarundi (Puma yagouaroundi). The composition of the five cats in 15 protected areas was compared grouped in three regions: the Moskitia, Caribbean and Central region with different life zones, ecosystems and altitudinal levels. Cat detection was evaluated by presence/absence in each protected area. Cluster analysis and non- metric multidimentional scaling were used to compare similarity between protected areas and species. The significant finding is the absence of the jaguar in protected areas in the Central Region of Honduras confirming local
} 
extirpation. Puma, ocelots, margay and the yaguarundi are present in the three regions. Comparing composition of cats between the three region using similarity analysis this presented significant difference between protected areas. The Central region showed a lower composition in cat structure that The Caribbean and Moskitia region. The Honduran Moskitia region showed a higher resilience to maintain the five cats.

Key words: Composition, conglomerates, non-metric multidimentional scaling (NMDS), analysis of similarity (ANOSIM), dissimilarity, Moskitia.

\section{INTRODUCCIÓN}

Honduras mantiene en su área geográfica cinco de los siete felinos registrados para Mesoamérica, siendo estos: el jaguar (Panthera onca), puma (Puma concolor), ocelote (Leopardus pardalis), yaguarundi (Puma yagouaroundí) y el caucel (Leopardus wiedii) (Marineros y Martínez, 1998). Los miembros de la familia felidae son los más especializados del orden de los carnívoros. Todas las especies son digitígrados, garras retráctiles, cabeza ancha y redondeada; dientes caninos excepcionalmente alargados y filosos, adaptaciones que los convierten en depredadores por excelencia (Goodwin, 1942). Los grandes predadores como los felinos funcionan como controladores en la dinámica de las poblaciones de presas y son indicadores de la integridad de los ecosistemas por su vulnerabilidad a los cambios bruscos en sus hábitats (Redford et al., 2005). Los felinos aun cuando son carismáticos y aceptados por su imagen de poder, agilidad y astucia la realidad con ellos es que son considerados dañinos por la mayoría de ganaderos o granjeros que han perdido más de una especie doméstica y que han sido atribuidas a estos depredadores (Amit et al., 2009). Las mayores amenazas a las que están sometidas estas especies en Honduras son; el avance de la frontera agrícola y como consecuencia la pérdida de hábitat; la cacería (felinos y las especies presas alimento de estos), colecta y tráfico de fauna (Secaira, 2013). Las diferentes amenazas así como el comportamiento natural hacen que los felinos sean especies de difícil observación (Maffei et al., 2002, Maffei et al., 2005). En Honduras se han implementado diferentes métodos para el monitoreo de ma- míferos grandes y medianos entre los que podemos mencionar: trampa cámara, rastros en transectos (huellas, pelos, eses), consultas y avistamientos directos. Entre los métodos que han resultado más eficaces para documentar la presencia de felinos en Honduras han sido las trampas cámara, minimizando el sesgo en su identificación, sin embargo para efectos de este análisis se incorporan muestreos en transectos y comunicaciones personales que complementan los registros fotográficos de felinos en dichas áreas protegidas. Estos muestreos han sido realizados en diferentes ecosistemas, gradientes altitudinales y con diferentes esfuerzos de muestreo en cada una de las 15 áreas con datos desde el año 2006 al 2013 registrando la ausencia o presencia de la especie. En Honduras existen 91 áreas protegidas decretadas legalmente a partir del año 1980, y represen$\tan 30 \%$ del territorio nacional (DAPVs, 2005). El presente análisis tiene como objetivo conocer las tendencias de variación en la composición de la familia Felidae en 15 áreas protegidas de Honduras, usando análisis multivariados de ordenamiento por similitud representados en un dendrograma y diagrama de dispersión multidimensional para su interpretación, al vincular ambos resultados en el análisis (Clarke, 1993; Legendre y Legendre, 1998). Esto permitirá estar al tanto de la situación actual de los felinos en 15 áreas protegidas donde se asume están declinando rápidamente. Este trabajo pretende llamar la atención de autoridades encargadas de las áreas protegidas y vida silvestre de Honduras con la intensión de poder generar y motivar acciones concretas y de impacto en pro de su conservación estas especies en el país. 


\section{MATERIALES Y MÉTODOS}

\section{Sitio de Estudio}

Los registros para este análisis provienen de diferentes informes de monitoreo biológico de instituciones del gobierno de Honduras, proyectos de conservación privados, organizaciones no gubernamentales de co-manejo de áreas protegidas, publicaciones de artículos en revistas científicas y comunicaciones personales. El análisis incluye 15 áreas protegidas en donde se han realizado monitoreos o estudios de manera puntual o sistemática. Estas 15 áreas protegidas tienen sus propias características entre las que podemos mencionar: presencia institucional para su administración (co-manejo), zonas de vida, el tipo de ecosistemas predominante, el gradiente altitudinal, topografía, tamaño del área. Para efectos de comparar la composición de los cinco felinos las áreas naturales protegidas se agruparon en tres regiones diferenciadas por sus zonas de vida, ecosistemas predominantes y rangos altitudinales, siendo estos: La región del Caribe o bosques del litoral Caribe; la cual se localiza en la zonas de vida del bosque húmedo tropical (bh-T), bosque muy húmedo sub tropical (bmhST), y el bosque muy húmedo montano bajo (bmh-MB) con rangos altitudinales de 200-2200 msnm con predominancia de los ecosistemas de bosques latifoliado nublado y latifoliado de tierras bajas, bosques mixtos (Pinus caribaea y Quercus sp), bosques de pino (Pinus caribaea), humedales y playa del Caribe. Las áreas protegidas que la conforman son: El Parque Nacional (PN) Jeannette Kawas, El Refugio de Vida Silvestre (RVS) de Texiguat, EI PN de Pico Bonito, EI PN Nombre de Dios. La región de la Moskitia hondureña perteneciente a la zona de vida del bosque húmedo tropical (bh-T) y el bosque muy húmedo subtropical (bmh-ST) con rangos altitudinales de 10-1000 msnm, con predominancia de los ecosistemas del bosque latifoliado de tierras bajas y las sabanas de pino (Pinus caribaea). Las áreas protegidas que la conforman incluye: La Reserva Biológica (RB) propuesta de Rus Rus, El PN Patuca, La Reserva de la Biósfera (RBio) Tawahka Asagni y La RBio Río Plátano. La región Central o región montañosa perteneciente a la zonas de vida del bosque húmedo subtropical (bh-ST) y el bosque muy húmedo montano bajo (bmh-MB) con rangos altitudinales de 600-2800 msnm, con predominancia de los ecosistemas del bosques latifoliado nublado, bosques mixtos de pino encino (Pinus oocarpa y Quercus sp) y los bosques de pino (Pinus $s p$ ) la conforman: El PN La Tigra, La RB El Chile, RB Misoco, RVS La Muralla, EI PN Sierra de Agalta, EI PN Cerro Azul Meámbar y el PN Montaña de Celaque. (ver Figura 1 y Tabla 1; Holdrige, 1971; Mejía y House, 2001).
Figura 1. Mapa de las áreas protegidas de Honduras agrupadas en las tres regiones; Caribe, Moskitia y Central (Fuente: DAPvs, 2005).

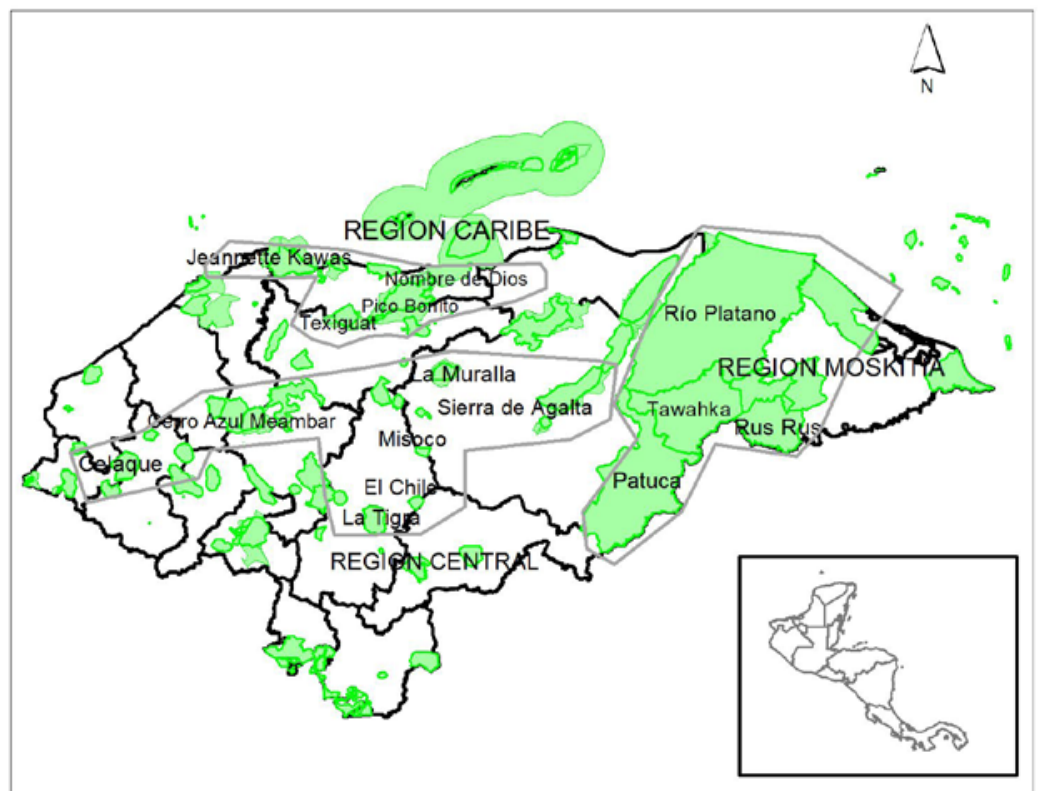


Tabla 1. Áreas protegidas agrupadas por región, con sus zonas de vida, ecosistemas predominantes, rangos altitudinales, tamaño en hectáreas, esfuerzo de muestreo noches cámara y el método utilizado para registrar la ausencia y presencia de los cinco felinos por área protegida.

\begin{tabular}{|c|c|c|c|c|c|c|}
\hline Región Central & Zona de Vida & $\begin{array}{l}\text { Ecosistemas } \\
\text { predominantes }\end{array}$ & $\begin{array}{l}\text { Rangos de } \\
\text { altitud msnm }\end{array}$ & $\begin{array}{l}\text { Tamaño en } \\
\text { ha }\end{array}$ & $\begin{array}{l}\text { Esfuerzo de } \\
\text { muestreo N/C }\end{array}$ & Métodos utilizados \\
\hline PN La Tigra & (bh-ST, bmh-MB) & $\begin{array}{l}\text { Latifoliado } \\
\text { nubloso, mixto } \\
\text { y pino }\end{array}$ & $800-2000$ & 24,341 & 2415 & $\begin{array}{l}\text { TC, huellas en } \\
\text { transectos }\end{array}$ \\
\hline $\begin{array}{l}\text { PN Sierra de } \\
\text { Agalta }\end{array}$ & (bh-ST, bmh-MB) & $\begin{array}{l}\text { Latifoliado } \\
\text { nubloso y mixto }\end{array}$ & $1000-2000$ & 51,793 & 390 & $\begin{array}{l}\mathrm{TC} \text {, huellas en } \\
\text { transectos }\end{array}$ \\
\hline PN CA Meámbar & (bh-ST, bmh-MB) & $\begin{array}{l}\text { Latifoliado } \\
\text { nubloso y mixto }\end{array}$ & $600-1600$ & 31,339 & 380 & $\begin{array}{l}\mathrm{TC} \text {, huellas en } \\
\text { transectos }\end{array}$ \\
\hline RVS La Muralla & (bh-ST, bmh-MB) & $\begin{array}{l}\text { Latifoliado } \\
\text { nubloso y mixto }\end{array}$ & $800-1600$ & 24,626 & 210 & $\begin{array}{l}\text { TC, huellas en } \\
\text { transectos }\end{array}$ \\
\hline RB El Chile & (bh-ST, bmh-MB) & $\begin{array}{l}\text { Latifoliado } \\
\text { nubloso y mixto }\end{array}$ & $800-1400$ & 6,453 & 810 & $\begin{array}{l}\mathrm{TC} \text {, huellas en } \\
\text { transectos }\end{array}$ \\
\hline RB Misoco & (bh-ST, bmh-MB) & $\begin{array}{l}\text { Latifoliado } \\
\text { nubloso y pino }\end{array}$ & $1200-1800$ & 4,572 & 600 & $\begin{array}{l}\text { TC, huellas en } \\
\text { transectos }\end{array}$ \\
\hline PN Celaque & (bh-ST, bmh-MB) & Mixto y pino & $800-2200$ & 26,631 & 210 & $\begin{array}{l}\text { TC, huellas en } \\
\text { transectos }\end{array}$ \\
\hline Total & & & & 169,755 & & \\
\hline \multicolumn{7}{|l|}{ Región Moskitia } \\
\hline PN Patuca & (bh-T, bmh-ST) & $\begin{array}{l}\text { Latifoliado de } \\
\text { tierras bajas }\end{array}$ & $600-1800$ & 376,447 & 585 & $\begin{array}{l}\mathrm{TC} \text {, huellas en } \\
\text { transectos }\end{array}$ \\
\hline RBio Río Plátano & (bh-T, bmh-ST) & $\begin{array}{l}\text { Latifoliado de } \\
\text { tierras bajas }\end{array}$ & $10-1400$ & 833,617 & 2857 & $\begin{array}{l}\mathrm{TC} \text {, huellas en } \\
\text { transectos }\end{array}$ \\
\hline RBio Tawahka & (bh-T, bmh-ST) & $\begin{array}{l}\text { Latifoliado de } \\
\text { tierras bajas }\end{array}$ & $600-1000$ & 252,058 & 2596 & $\begin{array}{l}\mathrm{TC} \text {, huellas en } \\
\text { transectos }\end{array}$ \\
\hline RB RusRus & (bh-T, bmh-ST) & $\begin{array}{l}\text { Latifoliado de } \\
\text { tierras bajas y } \\
\text { sabana de pino }\end{array}$ & $100-400$ & 116,349 & 4220 & $\begin{array}{l}\text { TC, huellas en } \\
\text { transectos }\end{array}$ \\
\hline Total & & & & 1578,470 & & \\
\hline \multicolumn{7}{|l|}{ Región Caribe } \\
\hline PN Pico Bonito & $\begin{array}{l}\text { (bh-T, bmh-ST, } \\
\text { bmh-MB) }\end{array}$ & $\begin{array}{l}\text { Latifoliado } \\
\text { nubloso, } \\
\text { latifoliado de } \\
\text { tierras bajas, } \\
\text { mixto y pino }\end{array}$ & $200-2200$ & 107,107 & 4329 & $\begin{array}{l}\text { TC, huellas en } \\
\text { transectos }\end{array}$ \\
\hline $\begin{array}{l}\text { PN Jeannette } \\
\text { Kawas }\end{array}$ & $\begin{array}{l}\text { (bh-T, bmh-ST, } \\
\text { bmh-MB) }\end{array}$ & $\begin{array}{l}\text { Latifoliado } \\
\text { inundado, playa }\end{array}$ & $10-200$ & 79,382 & 2520 & $\begin{array}{l}\text { TC, huellas en } \\
\text { transectos }\end{array}$ \\
\hline RVS Texíguat & $\begin{array}{l}\text { (bh-T, bmh-ST, } \\
\text { bmh-MB) }\end{array}$ & $\begin{array}{l}\text { Latifoliado } \\
\text { nubloso y mixto }\end{array}$ & $600-2000$ & 29,764 & 555 & $\begin{array}{l}\text { TC, huellas en } \\
\text { transectos }\end{array}$ \\
\hline $\begin{array}{l}\text { PN Nombre de } \\
\text { Dios }\end{array}$ & $\begin{array}{l}\text { (bh-T, bmh-ST, } \\
\text { bmh-MB) }\end{array}$ & $\begin{array}{l}\text { Latifoliado } \\
\text { nubloso, } \\
\text { latifoliado de } \\
\text { tierras bajas }\end{array}$ & 200-1800 & 30,281 & 360 & $\begin{array}{l}\text { TC, huellas en } \\
\text { transectos }\end{array}$ \\
\hline Total & & & & 246,534 & & \\
\hline
\end{tabular}




\section{Análisis de la información}

Para el presente análisis se revisaron los diferentes informes técnicos elaborados por las diferentes instituciones y organizaciones responsables de cada uno de los monitoreos. En vista de que cada monitoreo fue realizado con diferente métodos y esfuerzo de muestreo solamente se registró para este análisis la ausencia o presencia de cada una de las especies presente en las áreas protegidas. Como evidencia de registro se tomaron las fotocapturas, registros de huellas y comunicaciones personales con técnicos y personas de las localidades de las áreas analizadas. Se construyó una matriz principal binomial en Excel, con uno (1) para el registro de presencia y cero (0) para su ausencia en donde las filas representan las áreas protegidas y las columnas las especies con el nombre común, por ejemplo; $P$. onca (jaguar), $P$. concolor (puma), L. pardalis (ocelote), L. wiedii (caucel), P. yagouaroundi (yaguarundi). Se utilizó el análisis de conglomerados para conocer la relación de similitud entre áreas protegidas por la presencia de sus especies, este método se basa en distancias multivariadas agrupadas por pares (Gottelli y Ellisson, 2004). Así mismo como análisis complementario al conglomerado se realizó un análisis no métrico multidimensional (NMDS) que muestra gráficamente en un plano dimensional la relación de la similitud de las especies presentes por área protegida mostrando las agrupaciones entre las áreas (Clarke, 1993; Harper, 1999). El NMDS mantiene los rangos de similitud entre la composición de las especies y las áreas, mostrando agrupaciones en el grafico por la ausencia o presencia de las especies. Para evaluar la similitud o disimilitud por la presencia/ausencia de felinos entre regiones se utilizó el Análisis de Similitud (ANOSIM), el cual es un análisis multi-variado no paramétrico que da un valor $\mathrm{R}$ que indican disimilitud significativa o no entre grupos. Para los tres análisis anteriores se usó el Coeficiente de Similitud de Jaccard para datos binarios (Clarke, 1993; Legendre y Legendre, 1998). Se utilizó el programa estadístico PAST 1.94b (Paleontological Statistc) desarrollado por (Hammer y
Harper, 2006) para el análisis multivariado en la paleontología y ecología.

\section{RESULTADOS}

Los resultados muestran a través del dendrograma basándose en la ausencia/presencia y la similaridad de las especies presentes en las áreas protegidas cuatro conglomerados. Un primer conglomerado del $100 \%$ de similitud de especies siendo las áreas: El PN Patuca, La RB de Rus Rus, La RBio Tawahka Asagni, La Bio del Río Plátano, PN Sierra de Agalta, PN Nombre de Dios y el PN Pico Bonito. Un segundo conglomerado que contiene EI PN Cerro Azul Meámbar con el $80 \%$ de similitud con el primer conglomerado seguido del PN La Tigra y El PN Celaque con un $65 \%$ de similitud al primer conglomerado. Un tercer conglomerado que agrupa RVS La Muralla, La RB de El Chile y Misoco con un $65 \%$ de similitud con respecto al primer conglomerado y el cuarto conglomerado que igual registra un $65 \%$ de similitud con respecto al primer conglomerado y diferenciándose del tercer conglomerado por la presencia de diferentes especies con igual número (ver figura 2).

Al usar el NMDS para complementar el análisis de similitud de los resultados del dendrograma (Field et al., 1982) se observa las 15 áreas protegidas agrupadas en los cuatro grupos basados en la presencia ausencia de las cinco especies de felinos presentes en cada una de las áreas. Esto muestra la correspondencia entre el dendrograma y el análisis no métrico multidimensional (ver figura 3 y figura 4).

El NMDS obtuvo un valor de estrés de 0.173 que representa una agrupación no al azar, basada en los rangos de similaridad de las especies de felinos en las áreas protegidas (Kruskal and Wish, 1978; Clarke, 1993). El análisis de similitud (ANOSIM) entre las tres regiones presento un valor de $\mathrm{R}=0.30$ el cual se interpreta que existe una disimilitud entre las tres regiones comparadas (Clarke, 1993) La región Central mostró menor composición que la región del Caribe y la Moskitia. La región de la Moskitia hondureña registró la presencia de las cinco 


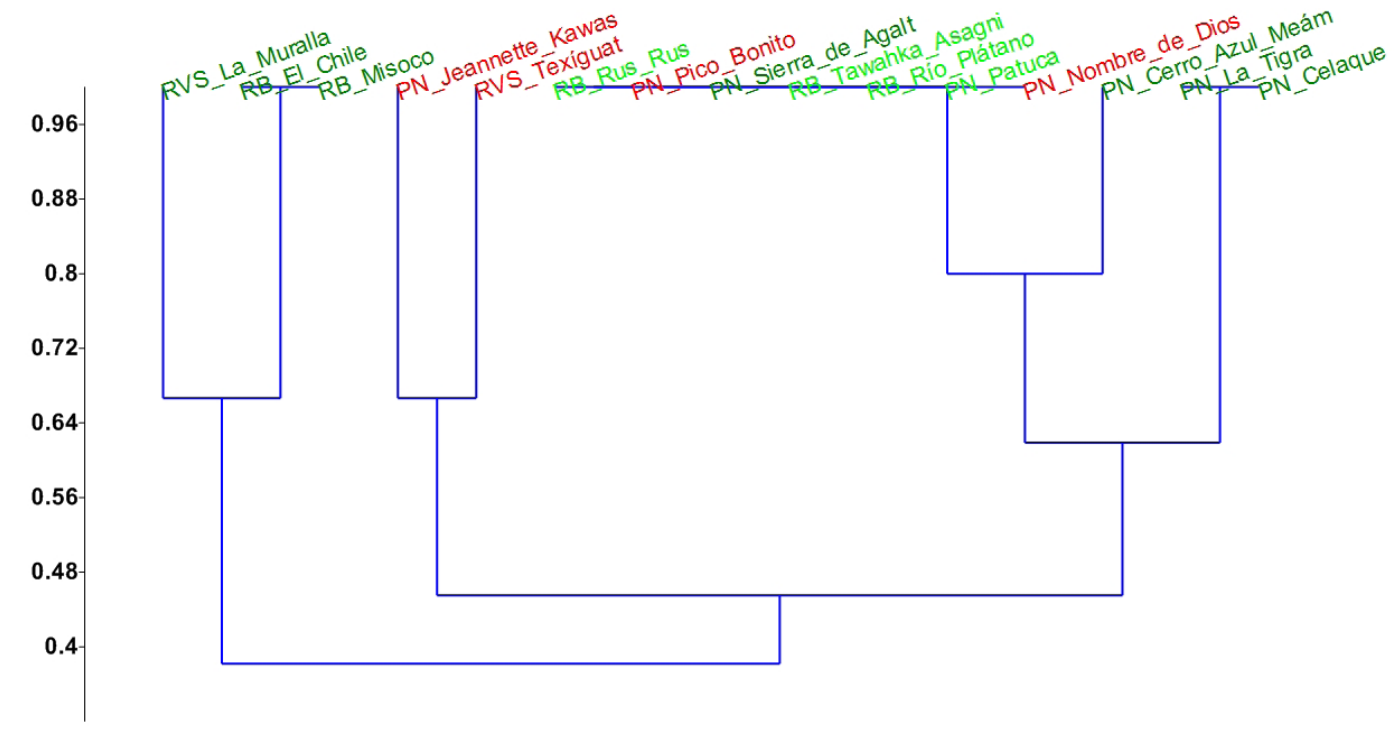

Figura 2. Dendrograma donde se observa la formación de los cuatro grupos por área protegida basados en los rangos de similitud de las especies de felinos presentes en las áreas protegidas.

Figura 3. Gráfico de Escalamiento Multidimensional No Métrico (NMDS) donde se agrupan las áreas protegidas por la similitud de especies presentes, aquí se puede apreciar la región de la Moskitia y Caribe agrupan las cinco especies de felinos presentes en Honduras. Los vectores muestran la relación por similitud de las especies y las áreas protegidas.

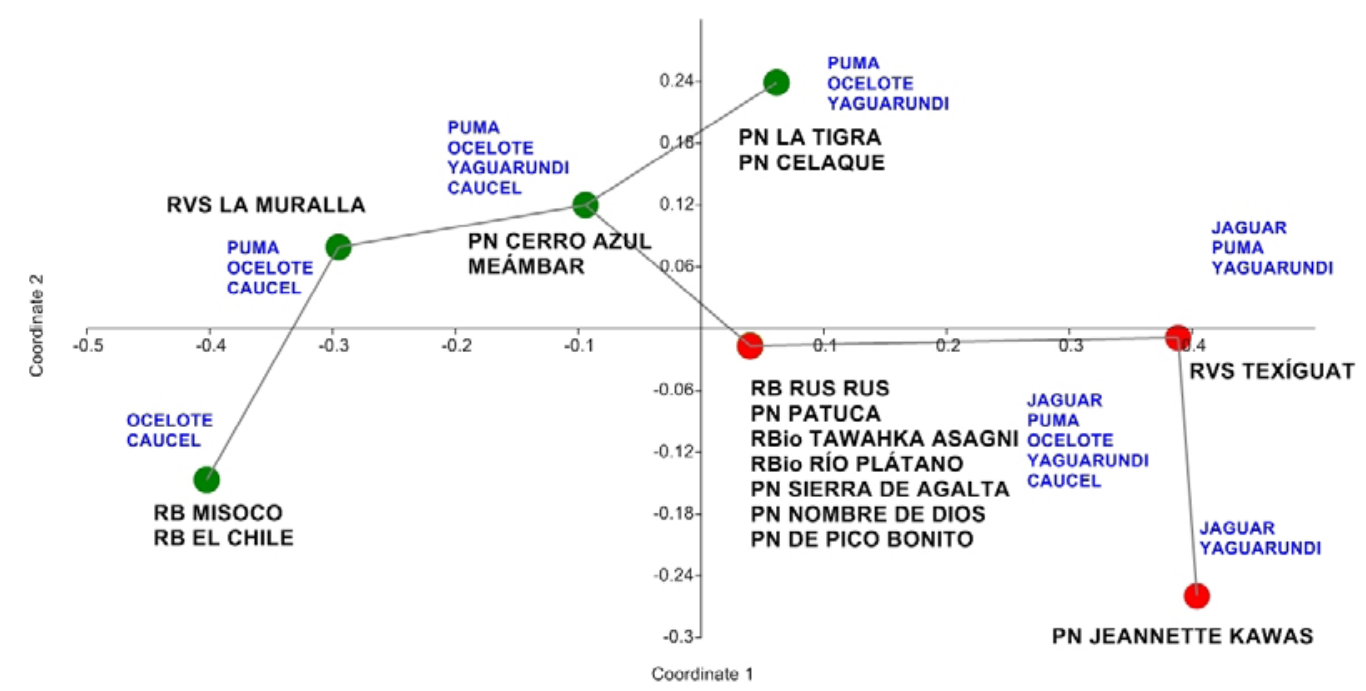




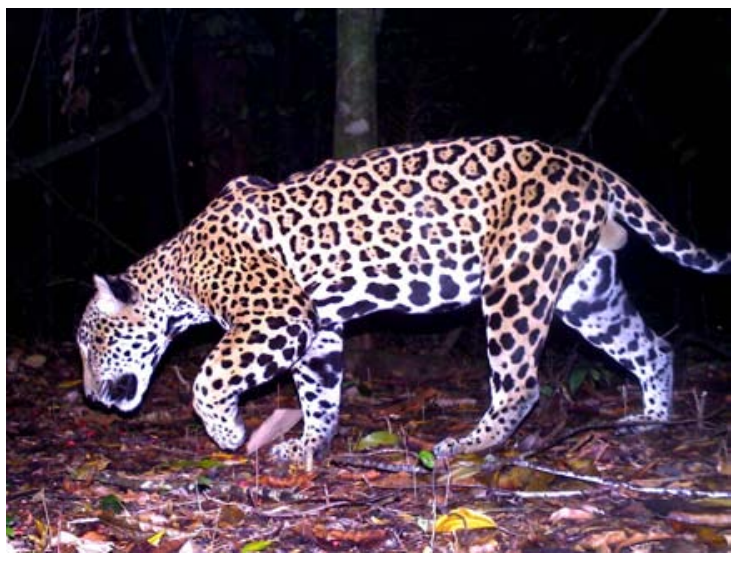
los cinco felinos que ocurren en Honduras. a)Jaguar (Panthera onca), b) Puma (Puma concolor), c) Ocelote (Leopardus pardalis), d) Yaguarundi (Puma yagouaroundi), e) Caucel (Leopardus wiedii). Cortesia: Fundación PHANTERA Honduras. b

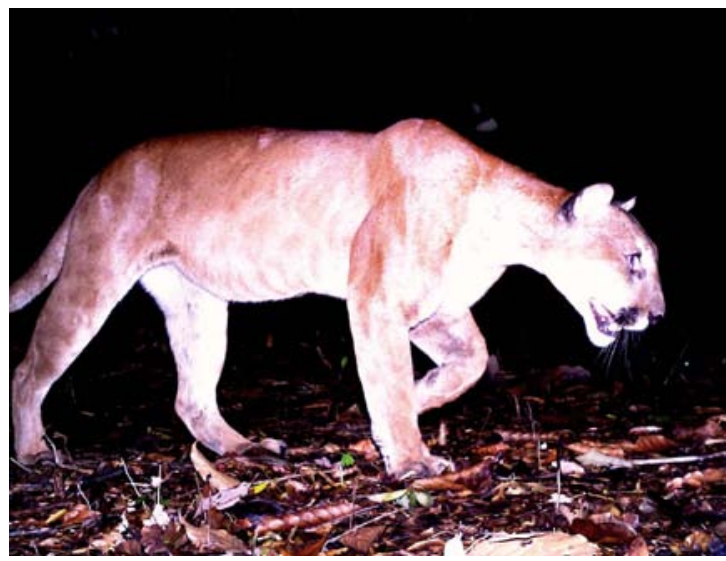

d

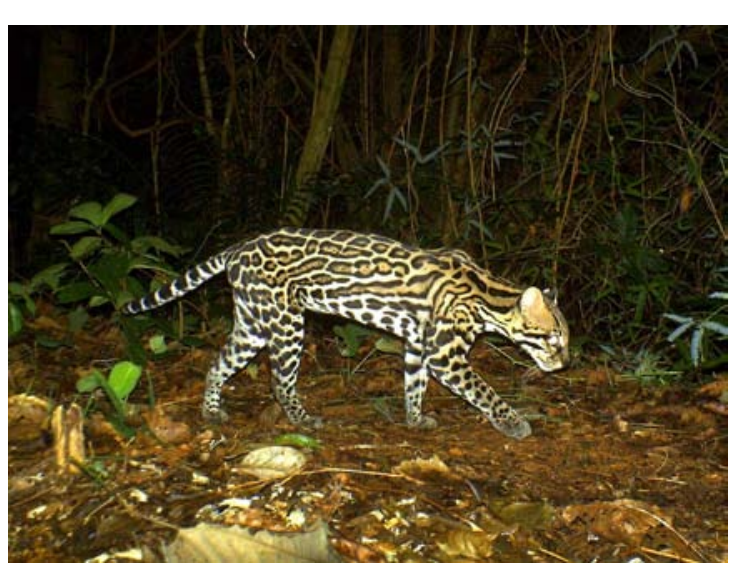

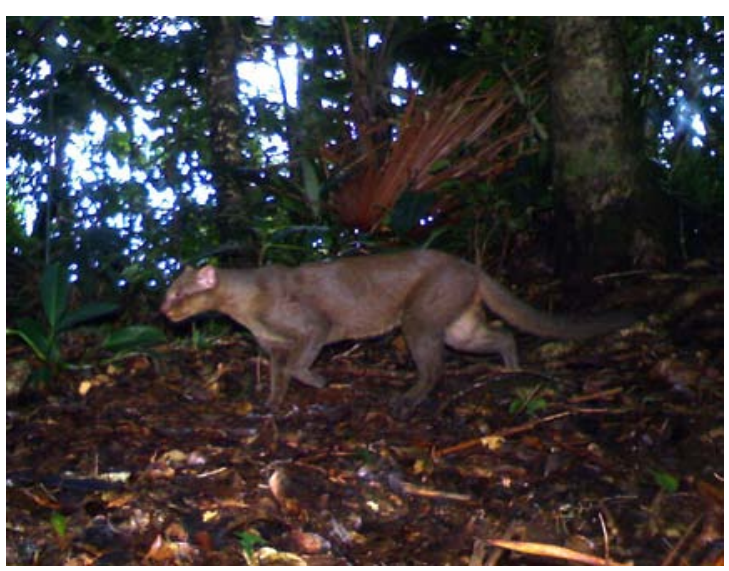

e

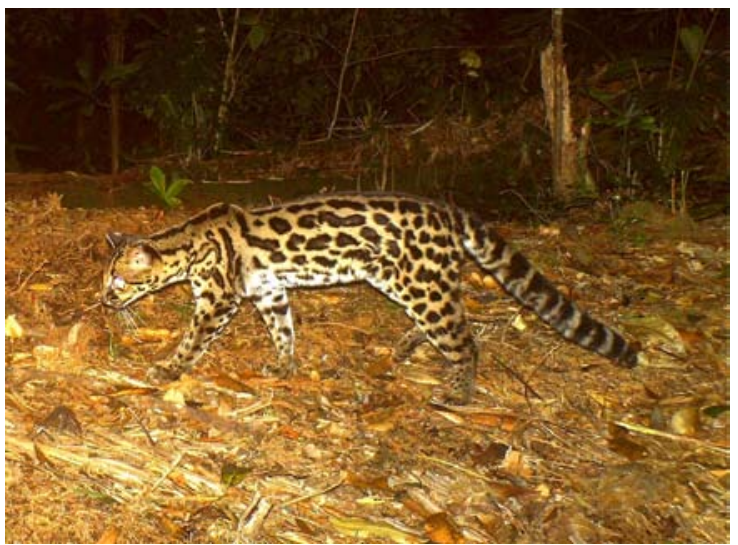


especies de felinos con la mejor composición, compartidas con el PN Sierra de Agalta de la región central y los parques nacionales de Pico Bonito y Nombre de Dios de la región del Caribe hondureño.

\section{DISCUSIÓN}

Los resultados muestran menor detección de los felinos en la región Central de Honduras. El más representativo es el jaguar, especie que estaba ampliamente distribuida en Honduras (Mora et al., en prensa) y que requiere de espacios territoriales y hábitats con un estado de conservación que le permitan alimentarse, reproducirse y refugiarse sin riesgos y a movilizarse a otros territorios en estas áreas protegidas. El puma se detecta en cuatro de las seis áreas protegidas de la región central, lo que nos indica mayor adaptabilidad a la presencia humana, ya que se le ha observado fuera los límites de áreas protegidas, movilizándose en los bosques mixtos y de pino entre áreas protegidas y en donde hay establecidas comunidades y sistemas productivos. Se asume que en áreas de menor tamaño los pumas utilizan estas áreas como sitios de paso o traslado, ya que son áreas relativamente pequeñas y con escaza conectividad con los ecosistemas de bosque mixto y pino entre otras áreas o sitios naturales protegidos. En la región del Caribe el puma y ocelote no se han detectado en el PN Jeannette Kawas después de largos periodos de muestreo con trampas cámara, este evento puedes ser debido a que el simpatrismo no se da por la competencia de alimento, espacio y refugio entre las especies, posiblemente debido al tamaño del área protegida y la extirpación de presas (Castañeda et al., 2013). En el PN Jeannette Kawas especies como el caucel la cual es una especie con hábitos más arborícolas que terrestres y el yaguarundi con actividades más a la periferia de los felinos mayores (Portillo-Reyes y Hernández, 2011) hacen más difícil su detección, especialmente cuando los monitoreos de trampas cámara son diseñados estrictamente para jaguares y pumas y los monitoreos de evidencias son diseñados para transectos con recorridos de día. La región de la Moskitia presenta las cinco especies de felinos, esta es la región en el país donde se localizan las áreas protegidas de mayor extensión territorial y enlazada de forma continua entre sí, formando masas boscosas continuas, ideales como hábitat. Esto ha permitido que la composición de felinos se mantenga con las cinco especies y que áreas como el PN Sierra de Agalta compartan estas especies, se asume que se debe a la relativa cercanía (entre $35-40 \mathrm{Km}$, lineales) a la región de la Moskitia que le ha favorecido con el intercambio de especies que se mueven entre estas áreas protegidas, mostrando aún la capacidad de conectar el paisaje que funciona como un corredor entre regiones y áreas. Aun cuando el avance de la frontera agrícola y cacería en la región de la Moskitia se da a una velocidad alarmante su resilencia permite albergar estas cinco especies permitiendo espacios para refugio, sitios de alimentación y reproducción (Secaira, 2012). Dos de las cuatro áreas protegidas de la región del Caribe EI PN Pico Bonito y el PN Nombre de Dios, muestran las mismas características que la región de la Moskitia, siendo el tamaño, el enlace de paisaje y condiciones del hábitat características para mantener las cinco especies de felinos. Esto ha favorecido la conservación de estas especies en sitios como el Caribe hondureño bajo fuertes presiones de urbanismo, ganadería y cacería.

De los hallazgos significativos es la ausencia del jaguar ( $P$. onca) la única especie de los cinco felinos que no se encuentra presente en las áreas protegidas de la región Central de Honduras donde posiblemente exista la extirpación del mismo (Mora et al., en prensa). El puma (P. concolor), el ocelote (L. pardalis), el caucel $(L$. wiedii) y el yaguarundí ( $P$. yagouaroundi) se registran en las tres regiones; Moskitia, Caribe y Central del país.

La pérdida de hábitat, la cacería de los felinos, así como las presas alimento de estos, son factores que están diezmando a los felinos en general (McNab and Polisar, 2002). Debido a esta problemática, se ha sugerido en los planes de conservación para las poblaciones de jaguar que este sea considerado como 
la especie sombrilla para las otras especies de felinos que se encuentran en estado de peligro, y por ende se hacen necesarias acciones para su conservación, tales como censos, manejo de factores limitantes y acciones en torno a la cacería (Nowell y Jackson, 1996; Matamoros et al., 1997; ICF, 2011). La ganadería es otra de las amenazas que genera el conflicto felino-hombre en los distintos frentes de colonización, por lo que enfrentar este problema con los actores involucrados es prioritario para la conservación de los felinos en Honduras (ICF, 2011).

Según el análisis de similitud (ANOSIM) las tres regiones muestran disimilitud entre las áreas protegidas, esto se debe a que la región de la Moskitia, por su extensión territorial de más de un millón de hectáreas ha permitido mantener una alta resilencia para conservar la presencia de las cinco especies de felinos y sus presas entre ellas ; el danto (Tapirus bairdii), el venado tilopo (Mazama temama), la jagüilla ( Tayassu pecari) dentro de sus límites geográficos (Portillo-Reyes y Hernández, 2011). En el caso del PN Pico Bonito de la región Caribe, donde se registran las cinco especies de felinos, esta se enlaza con el PN Nombre de Dios que forma un paisaje continuo entre las dos áreas protegidas permitiendo a los felinos y sus presas desplazarse en áreas de mayor extensión. Las áreas protegidas de la región Central registran de dos a cuatro especies de los felinos, estas son áreas relativamente de menor tamaño que las áreas protegidas de la región de la Moskitia y región Caribe (Tabla 1). Las áreas protegidas de la región Central se encuentran enlazadas parcialmente por ecosistemas de pino-encino y sistemas productivos. Se asume que está fragmentación entre áreas y la cacería de felinos en la región Central ha interrumpido el paso de felinos y otros mamíferos mayores llegando a la extirpación regional como es el caso del jaguar (Mora et al., en prensa-). La presencia de organizaciones de la sociedad civil (manejo compartido de ONG y el estado de Honduras) en las áreas protegidas, combinadas con las vastas extensiones territoriales, han sido fundamental para que las áreas protegidas de la Moskitia y la región del Caribe mantengan las cinco especies de felinos pese a la presión a las que están expuestas por las actividades humanas. Es importante diseñar mantener programas de monitoreo de mamíferos y sus abundancias ya que permitirá analizar datos cuantitativos cuyo soporte estadístico será más robusto para establecer tendencias más acertadas del estado de las poblaciones de estos felinos y sus presas. La preservación de los felinos en áreas protegidas es primordial para el bienestar de las poblaciones de mamíferos mayores y menores en los ecosistemas, especialmente para el bienestar humano que permanentemente se beneficia de los servicios y bienes proveídos por las áreas protegidas del país. La presencia de cualquiera de estas cinco especies puede motivar la gestión, conservación y protección de espacios naturales que benefician la colectividad y el bienestar para la biodiversidad y por ende al ser humano como ente primordial para proveer la sostenibilidad de los recursos naturales en el país.

\section{Agradecimientos}

Se agradece a los técnicos del Instituto de Conservación Forestal (ICF) por facilitar los informes de trampas cámara especialmente a Eduardo Rico, Rony Moreno y Marció Martínez. A las organizaciones privadas como PANACAM, PANAM, FPNAND, MAPANCE, PANTHERA, La cámara de Turismo de la Ceiba, LARECOTUR, ICADE, WCS, GIBH-BALAM, proyectos; MIRA/USAID Ecosistemas/PNUD, Moskitia/PNUD, TNC/UNAG que contribuyeron con la información de sus proyectos de monitoreo con trampas cámara.

\section{Literatura citada}

Amit, R., K. Rojas, L.D. Alfaro y E. Carrillo. 2009. Conservación de Felinos y sus Presas Dentro de Fincas Ganaderas. Informe Técnico. Programa Jaguar Icomvisuna. Heredia, Costa Rica. $100 \mathrm{p}$

Castañeda, F. Herrera, L. Pereira, S. 2013. Behaviour of Two Male Jaguar Scavenging on a Marine Dolphin in Honduras. Rev. Catnews 58 Spring.

Clarke, R.K. 1993. A Method of linking Multivariate Community Structure to Environmental Variables. Australian Journal of Ecology, 18(1):117-143.

DAPVS (Departamento de Áreas Protegidas y Vida Silvestre), 2005. Actualización del Plan Estratégico del Sistema Nacional de Áreas Protegidas de Honduras (SINAPH), 2006-2015. Proyecto Biodiversidad y Áreas 
Protegidas (PROBAP). Tegucigalpa Honduras. 75 p. 11

Field, J.G. Clarke, K.R. and Warwick, R.M. 1982. A Practical Strategy for Analysing Multispecies Distribution Patterns. Marine Ecology, Progress series, 8:37-52,

Gotelli, N. and Ellison, A. 2004. A Primer Ecological Statistic. 1er Edition. Sunderland, MA. Sinauer Associate, Inc.,

Goodwin, G.G. 1942. Mammals of Honduras. Bulletin of the American Museum of Natural History, 79:107-195.

Hammer, Ø. and Harper, D.A.T. 2006. Paleontological Data Analysis. Blackwell.

Harper, D.A.T. (ed.). 1999. Numerical Palaeobiology. John Wiley \& Sons.

Holdrige, L. 1971. Ecología basada en zonas de vida. Instituto Interamericano de Ciencias Agrícolas. IICA. 216 p.

ICF. 2011. Plan Nacional para la Conservación del Jaguar (Panthera onca). Promoviendo la convivencia Comunidad - Jaguar. Departamento de Vida Silvestre/Instituto Nacional de Conservación y Desarrollo Forestal, Áreas protegidas y Vida SilvestreProyecto Ecosistemas- Fundación Panthera. Tegucigalpa. 29p.

Kruskal J.B. and Wish M.1978. Multidimensional Scaling. Sage Publications, Beverly Hills, California.

Legendre, P. and Legendre L. 1998. Numerical Ecology. Second English edition. Amsterdam. Elsevier Science B.V.,
Maffei L, Noss A, Cuellar E y Rumiz D. 2005. Ocelot (Felis pardalis) population densities,

activity, and ranging behavior in the dry forests of eastern Bolivia: data from camera trapping. Journal of Tropical Ecology, 21:1-6.

Maffei L, Cuellar E y Noss A. 2002. Uso de trampas-cámara para la evaluación de mamíferos en el ecotono Chaco-Chiquitanía. Revista Boliviana de Ecología y Conservación Ambiental, 11: 55-65.

Marineros, L y Martínez, F. 1998. Guía de Campo de los Mamíferos Silvestres de Honduras. Tegucigalpa. Instituto Nacional de Ambiente y Desarrollo Matamoros Y, Aranda M, Ellis S, Wildt D y O Byers (editores). 1997. Informe final del Taller para el Manejo y la Conservación de los Félidos Mesoamericanos. CBSG/SSC/ UICN, Zoológico Nacional Simón Bolívar, San José, Costa Rica.

Mejía, T y House, P. 2002. Mapa De Ecosistemas Vegetales de Honduras. Manual de Consultas AFE/COHDEFOR. Proyecto PAAR. Tegucigalpa. 60p.

Mora, J., J. Polisar, H. Portillo y F. Castañeda. en prensa. Estado de conservación del jaguar (Panthera onca) en Honduras. en: El jaguar en el Siglo XXI: La Perspectiva Continental. (Medellín, R., J. A. de la Torre, H. Zarza, C. Chávez y G. Ceballos, eds.). Fondo de Cultura Económica, Instituto de Ecología, UNAM, México.

McNab, R; Polisar, J. 2002. Una metodología participativa para una estimación rápida de la distribución del jaguar en Guatemala. Pp. 73-90, en: El jaguar en el nuevo milenio. (Medellin, R., Equihua, C., Chetkiewicz, C., Crawshaw, P., Rabinowitz, A., Redford, K., Robinson, J., Sanderson, E., Taber, A., eds.). Fondo de Cultura Económica, Universidad Autónoma de México y Wildlife Conservation Society. México, MX.

Nowell, K and Jackson, P. 1996. Wild Cats: Status, survey and conservation action plan. UICN. Gland, CH. 382 p.

Portillo-Reyes $\mathrm{H}$ and Hernández J. 2011. Densidad del jaguar (Panthera onca) en Honduras: primer estudio con trampascámara en La Mosquitia hondureña. Revista Latinoamericana de Conservación, 2(1):4550.

Redford K. 2005. Introduction: How to value large Carnivorous Animals. Pp. 1-6, en: Large Carnivores and the conservation of Biodiversity (Ray, J., Redford, K., Steneck, R., Berger, J. eds.) . Island Press Washington, US.

Secaira, E. 2013. Análisis y síntesis de los 10 planes de conservación elaborados para las áreas protegidas de trabajo del proyecto ProParque. usaID ProParque. 